\title{
AIRWORTHINESS TESTS OF THE UAV STRUCTURE - FATIGUE ISSUES
}

\author{
Mirosław Rodzewicz \\ Warsaw University of Technology, Warsaw, Poland
}

\begin{abstract}
The paper presents investigations undertaken in order to support developing the UAV airworthiness requirements, in particular those concerning fatigue aspects. Knowledge about UAVS load spectra is of vital importance for fatigue issues. The results of preliminary research are presented in this paper. A description of the load spectrum processing is given, starting from filtering the input-data, and then either counting the load signal transfers or implementing the Rainflow Counting algorithm and presenting the results in the form of a transfer array or halfcycles array. This description is illustrated by three examples of flight tests of 4-meter class UAVs, designed at Warsaw University of Technology. They concern flights controlled in a manual and automatic way in order to show differences between the LSs for such control modes. Besides the $L S$ processing, a method for statistical analysis of load spectra from several flights is also presented to address the important problem of LS dispersion. Finally, there are shown selected tests of the UAV structure elements for which load spectra may have a crucial importance.
\end{abstract}

Keywords: UAV loads, load spectrum, manual control, autopilot.

\section{INTRODUCTION}

A significant increase in the applications of UAVs has been observed in the last two decades mostly in the military domain. At the same time the economical aspects have raised interest in the application of UAVs also for civilian purposes. The potential possibilities of such applications include: terrain monitoring during disasters, monitoring of forests and crops, monitoring of communication tracks, media or energy transfer lines etc. Undoubtedly, the use of UVAs would be cheaper than that of GA aircraft. The main obstacle here is the lack of law requirements concerning the use of air space by UAV aircraft. It is a challenge for the scientific community to make UAVs available for civilian purposes. This paper presents preliminary investigations related to strength and fatigue safety of the UAV structures. Those investigations were carried out under the UAVs development program conducted at Warsaw University of Technology under the leadership of prof. Zdobyslaw Goraj. The object of investigations was SAMONIT 1 - an UAV destined for from-air monitoring missions. The basic data of this aircraft are displayed in Fig. 1. 


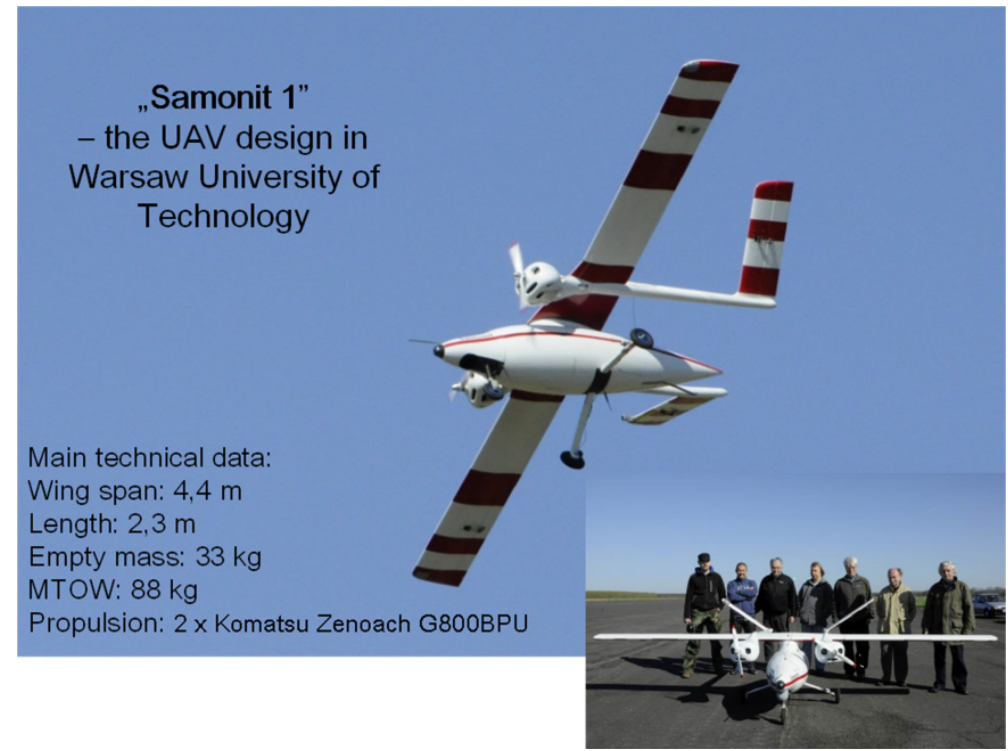

Fig. 1. SAMONIT 1 and its technical data

SAMONIT can be controlled either in a manual way (by a RC device) or in an automatic way by a MicroPilot L-2128 (Fig. 2). Attention was focused on the load spectrum induced by both ways of the UAV control. Knowledge about load spectrum is necessary to define fatigue durability requirements of the UAV-structure to be included in the UAV airworthiness regulations.

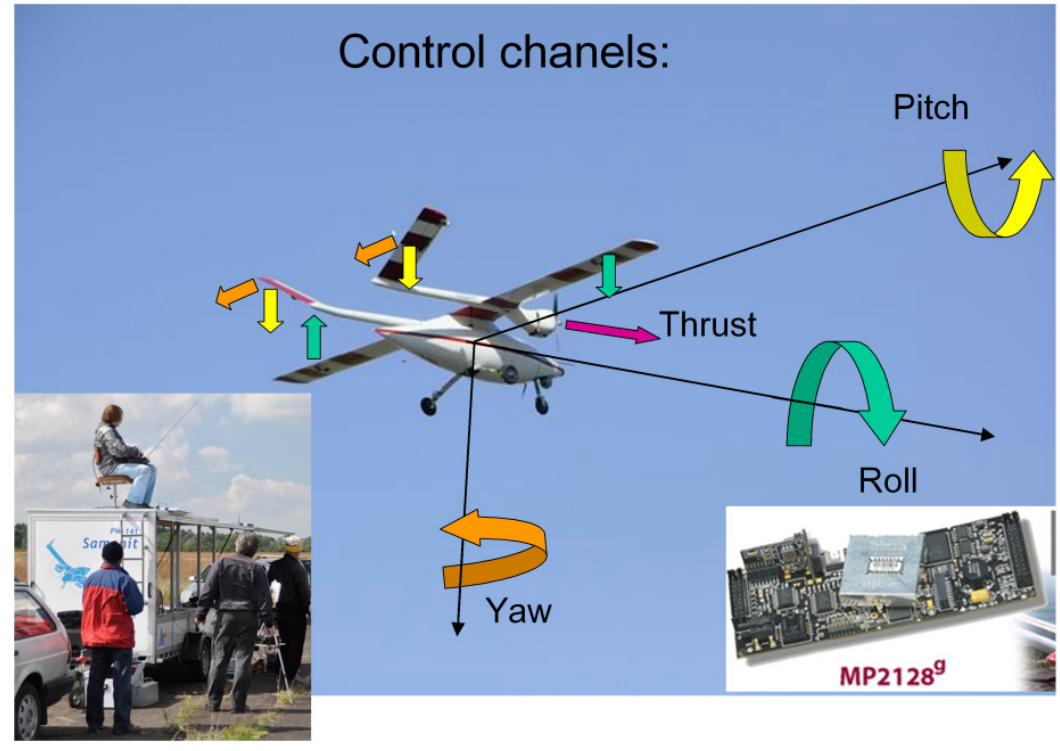

Fig. 2. SAMONIT 1 - control channels

Besides its main function of controlling the aircraft, the autopilot has anther useful feature of acquiring flight data (the total of 52 channels). Those data are of great importance for flight dynamics analysis including load spectrum derivations.

Figure 3 shows a screen-shot of the MicroPilot software for data-log viewing. The upper window contains some selected signals: airspeed, altitude and acceleration in the $z$-axis direction (i.e. perpendicular to the wing-plane), while the lower window contains the GPS-track. The analysis described in this paper focused on the $a_{z}$ acceleration signal because it may be easily recalculated into the load factor $n_{z}$ as a ratio of $a_{z} / g$, where $g$ is Earth's gravity. 


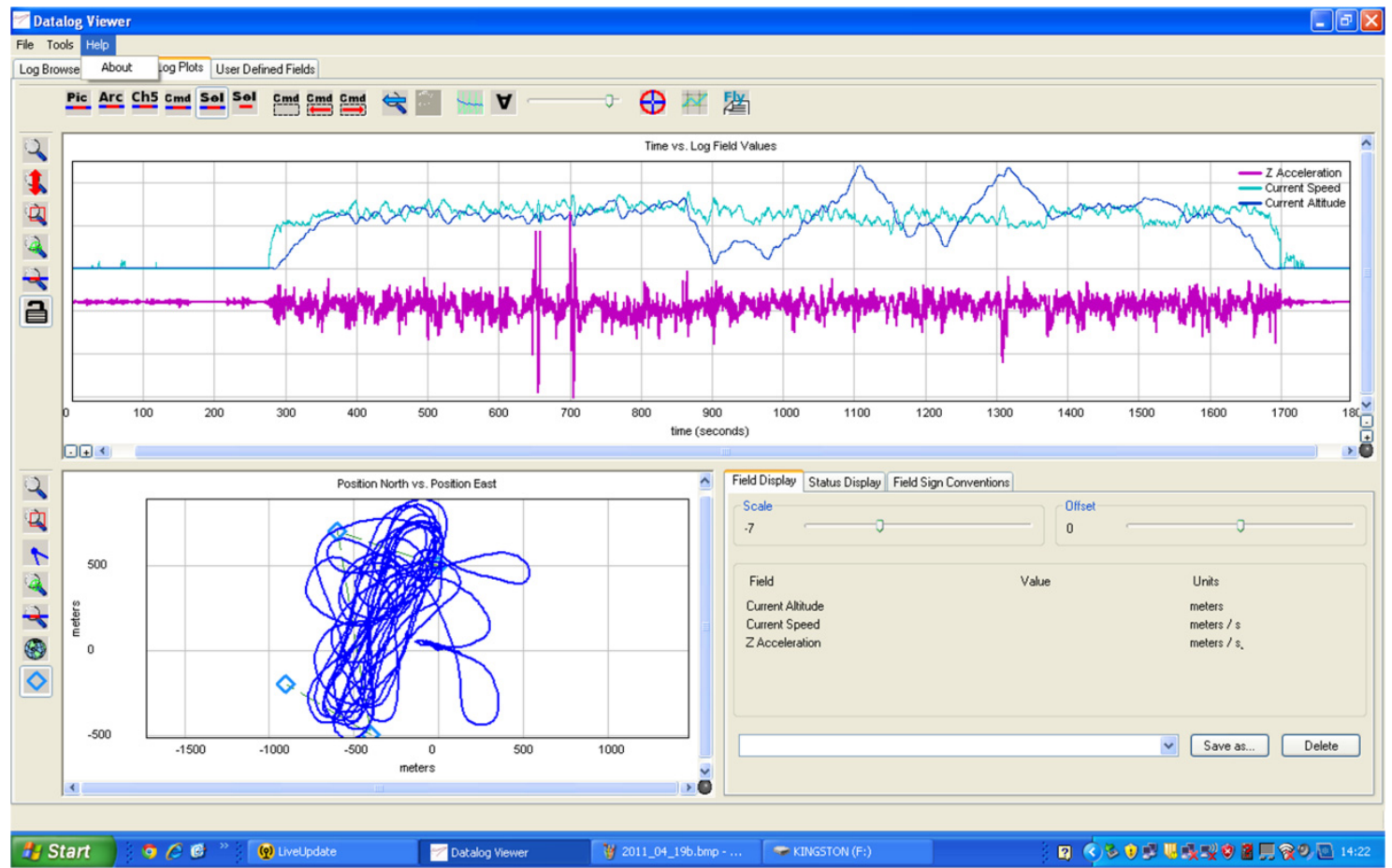

Fig. 3. Data-log Viewer-screen-shot of MicroPilot software

\section{TRANSFER ARRAYS AS A TOOL FOR LOAD-SIGNAL ANALYSIS}

As a tool for load signal analysis there were used Transfer and Half-Cycles arrays. The transfer arrays (T-arrays) contain information regarding number of signal transfers from each particular level to another level. The signal range in MicroPilot data-log is divided into 128 levels, however, for the purposes of the present investigations, the number of signal levels was reduced to 32 per range. Thanks to this simplification it was possible to apply the software tools previously used by the author in investigations of composite gliders' load spectra. There was also applied a standard correlation between $n_{z}$ and load level $L L$, i.e. $L L=3$ for maximum design value of the $n_{z}$, and $L L=31$ for minimum design value of the $n_{z}$.

To be able to count signal transfers a load signal should be first reduced to the form of a local extremes chain. Subsequently, either the algorithm shown in Fig. 4 or the Rainflow counting algorithm shown in Fig. 5 should be applied. The result obtained in the second case is a specific transfer array called a half-cycle array (HC-array). The important feature of a T-array and HCarrays is a so called operational zone (Fig. 4), i.e. a square envelope of all cells having non-zero values. This envelope defines the range of signal variability and is marked on the T- and HCarrays as a darker square. Operational zones of the T-array and $\mathrm{HC}$-array derived from the same signal have equal dimensions, but only $\mathrm{HC}$-arrays can be used in fatigue damage calculations because in a basic T-array does not register the resulting changes of signal levels after several transfers. 


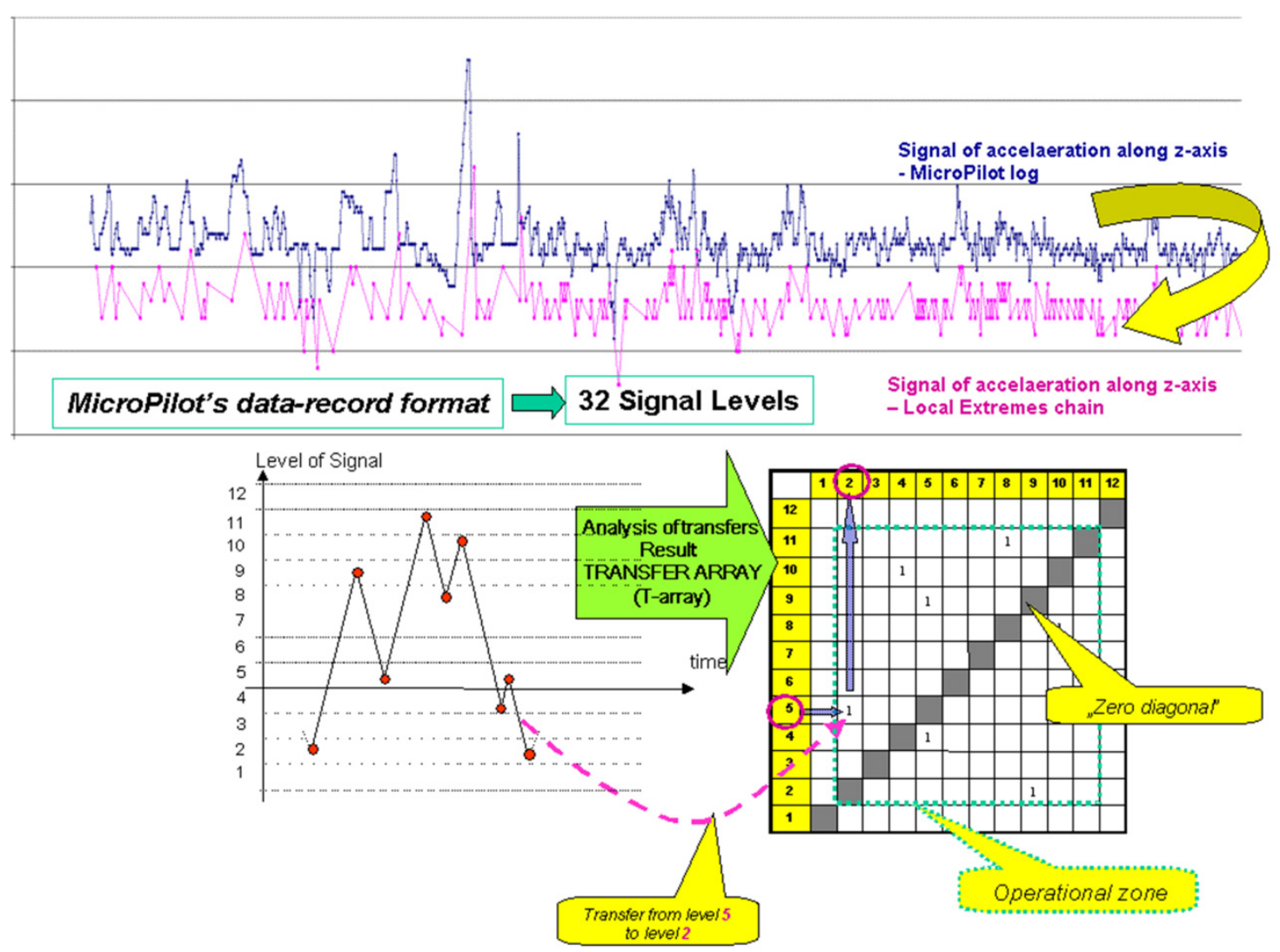

Fig. 4. Signal elaboration and Transfer-array determination

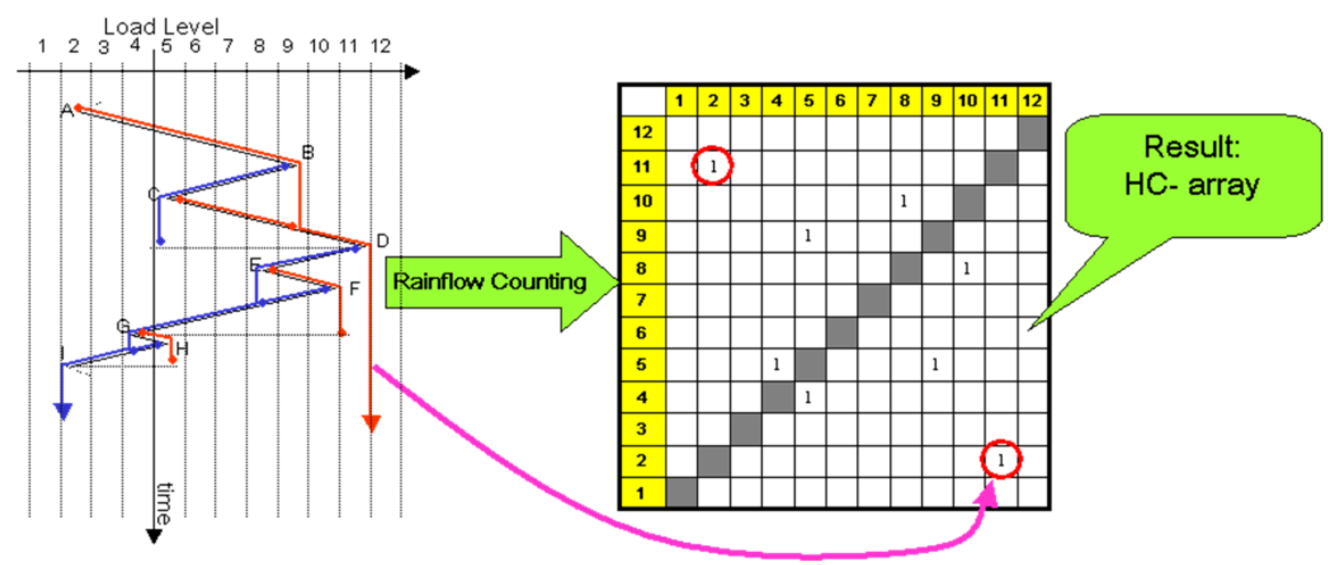

Fig. 5. Half-Cycle array as a result of applying Rainflow Counting algorithm

On the basis of the HC-arrays it is possible to create an incremental form of load spectra (Fig.6). A basic form of load spectrum presentation in the past, today an incremental form of load spectra has is of limited value for fatigue calculations, but still can be used for comparative analysis of different load spectra.

As an example there are shown incremental load spectra derived from the T-array and the HCarray obtained from the data-log. The flight considered was named Flight No.1 and is presented in Fig. 7. In this flight the UAV was controlled partly in an automatic and partly in a manual mode. The track length was about $41 \mathrm{~km}$ and the flight duration was about $950 \mathrm{~s}$. 


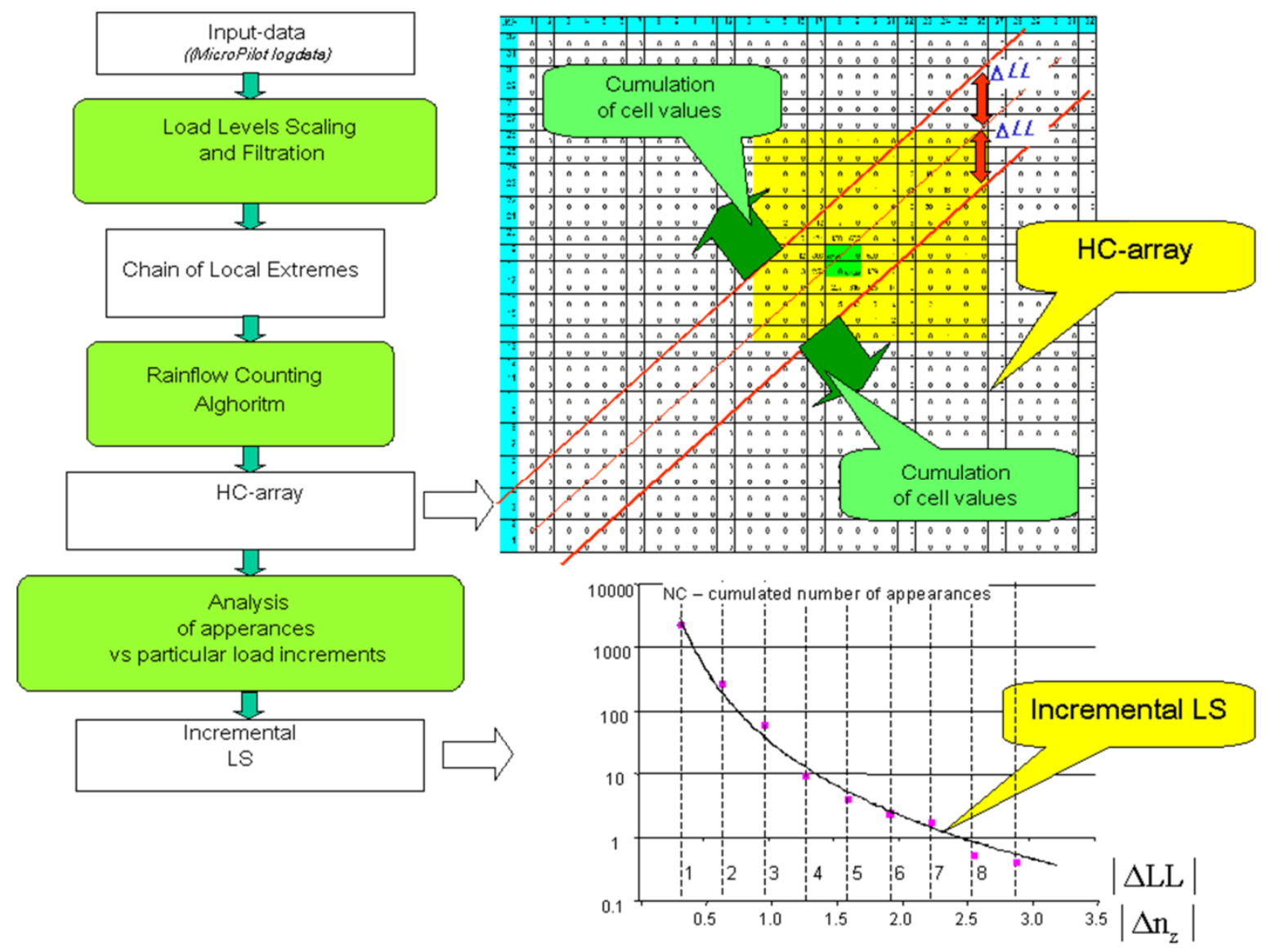

Fig. 6. The procedure of incremental load spectrum derivation

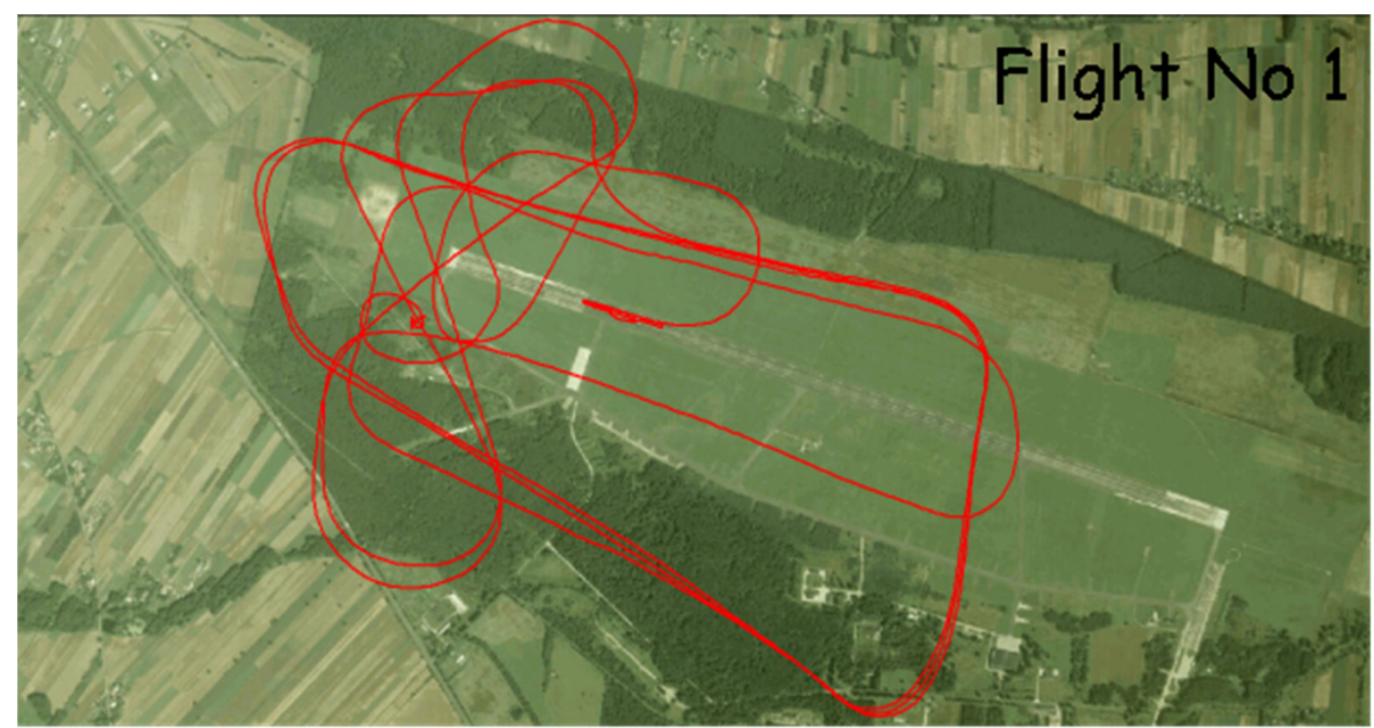

Fig. 7. Track of Flight No. 1

As it can be seen in Fig. 8, the maximum value of load increment is much lower in the case of applying the basic T-array algorithm that makes the reason why the Rainflow Algorithm is so important in fatigue calculations. 


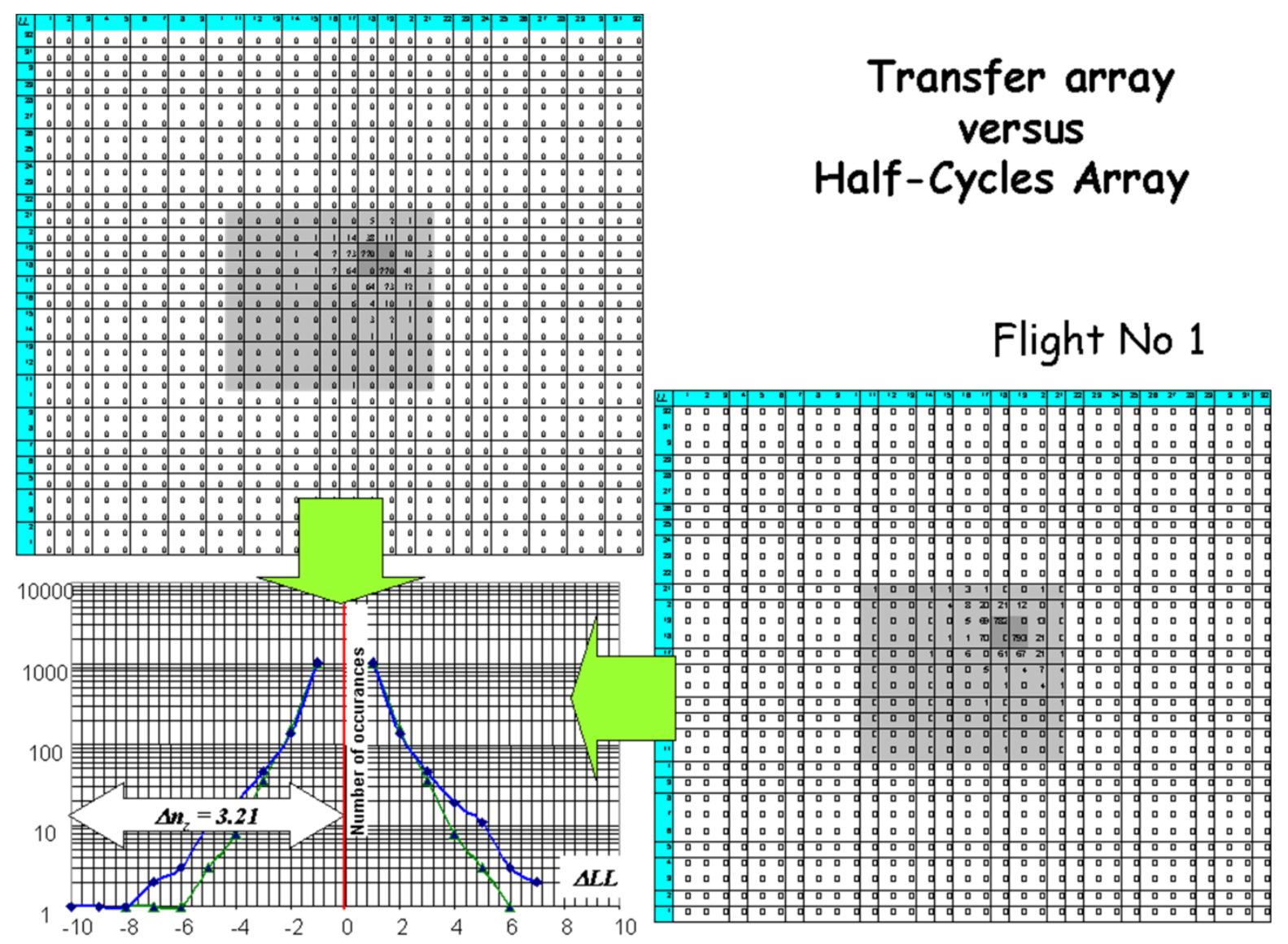

Fig. 8. T-array versus HC-array derived from the same signal

\section{LOAD SPECTRA FOR THE UAV CONTROLLED IN A MANUAL OR AUTOMATIC WAY}

The tools presented in the previous chapter were used for comparison of load spectra derived from the logs of two subsequent flights of SAMONIT 1: Flight No. 2 was made in a manual mode, while Flight No. 3 was mainly controlled by the autopilot. Both flights were made at the same airfield in similar weather conditions (Fig. 9). The times of flights were: 22 (Flight No. 2) and 17 minutes (Flight No. 3).

The HC-array from Flight No. 2 is shown in Fig. 10. The operational zone is larger here, and, interestingly, it contains the signal transfer between $10^{\text {th }}$ and $27^{\text {th }} L L$ (i.e. between $n_{z}=-1.71$ and $\left.n_{z}=3.75\right)$. It is a significant variation, bearing in mind the fact that the UAV did not make any aerobatic evolutions.

In the case of Flight No. 3 - controlled by the autopilot - the operational zone was much smaller (see Fig. 11). Those observations are consistent with the incremental load spectra shown in Fig. 12. The interesting feature of both curves is a similarity in the range of $L L \mid<7$.

The experiment leads to the conclusion that the autopilot mode produces a softer load spectrum than the manual mode of control does, but the number of flights being analyzed was too low to make any general conclusions. 

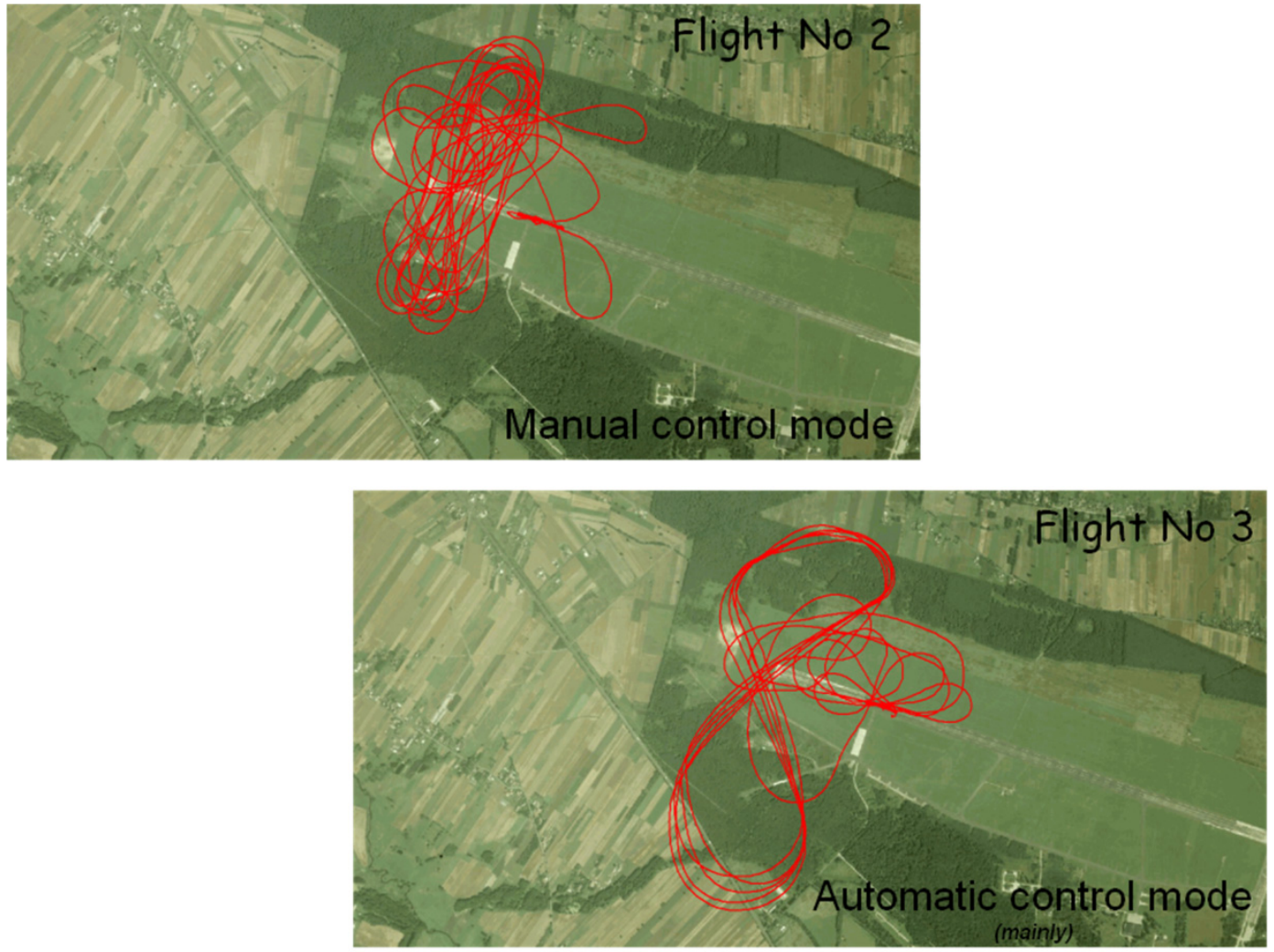

Fig. 9. Tracks of flights No. $2 \&$ No. 3

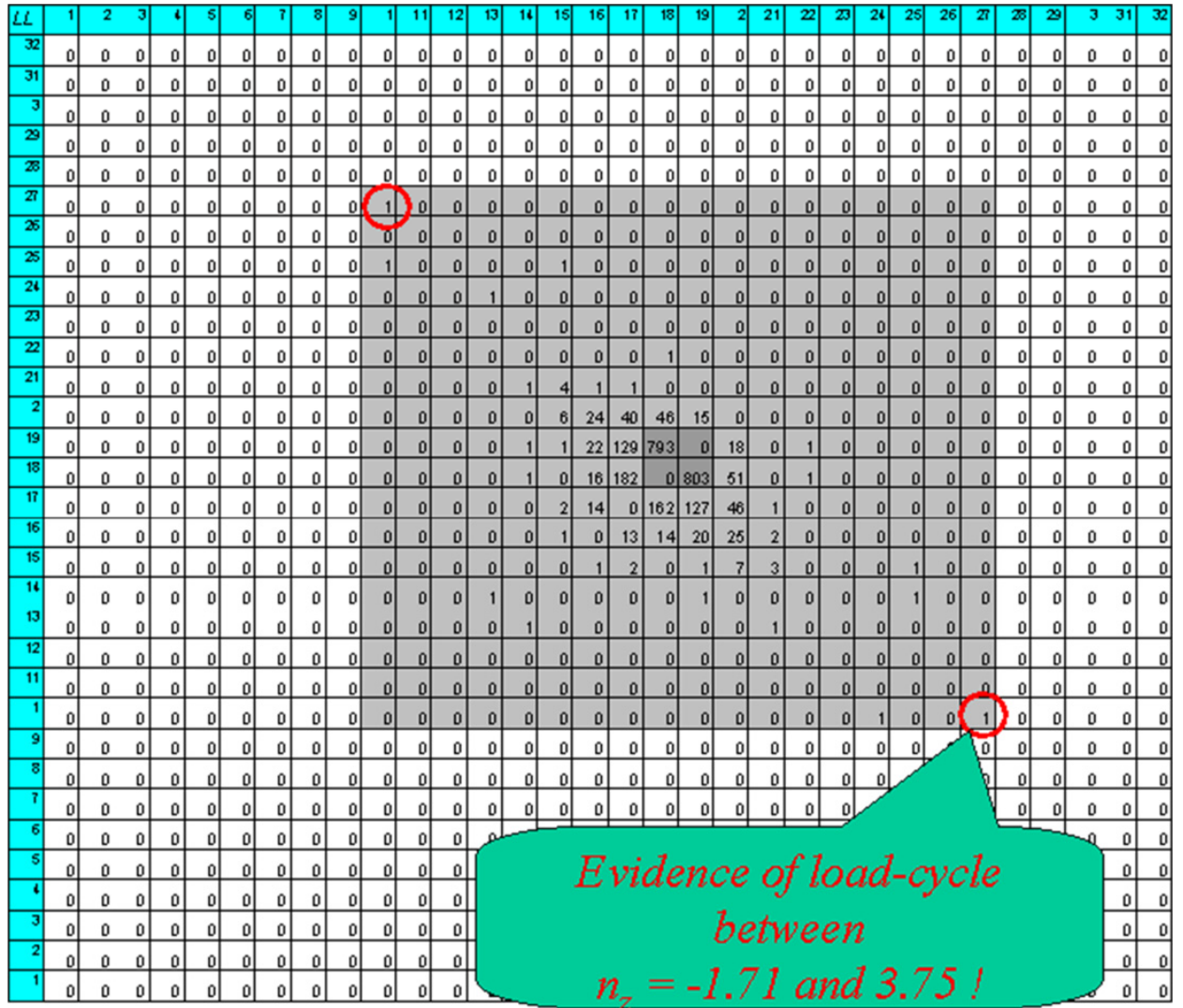

Fig. 10. HC-array from Flight No. 2 (manual control mode) 


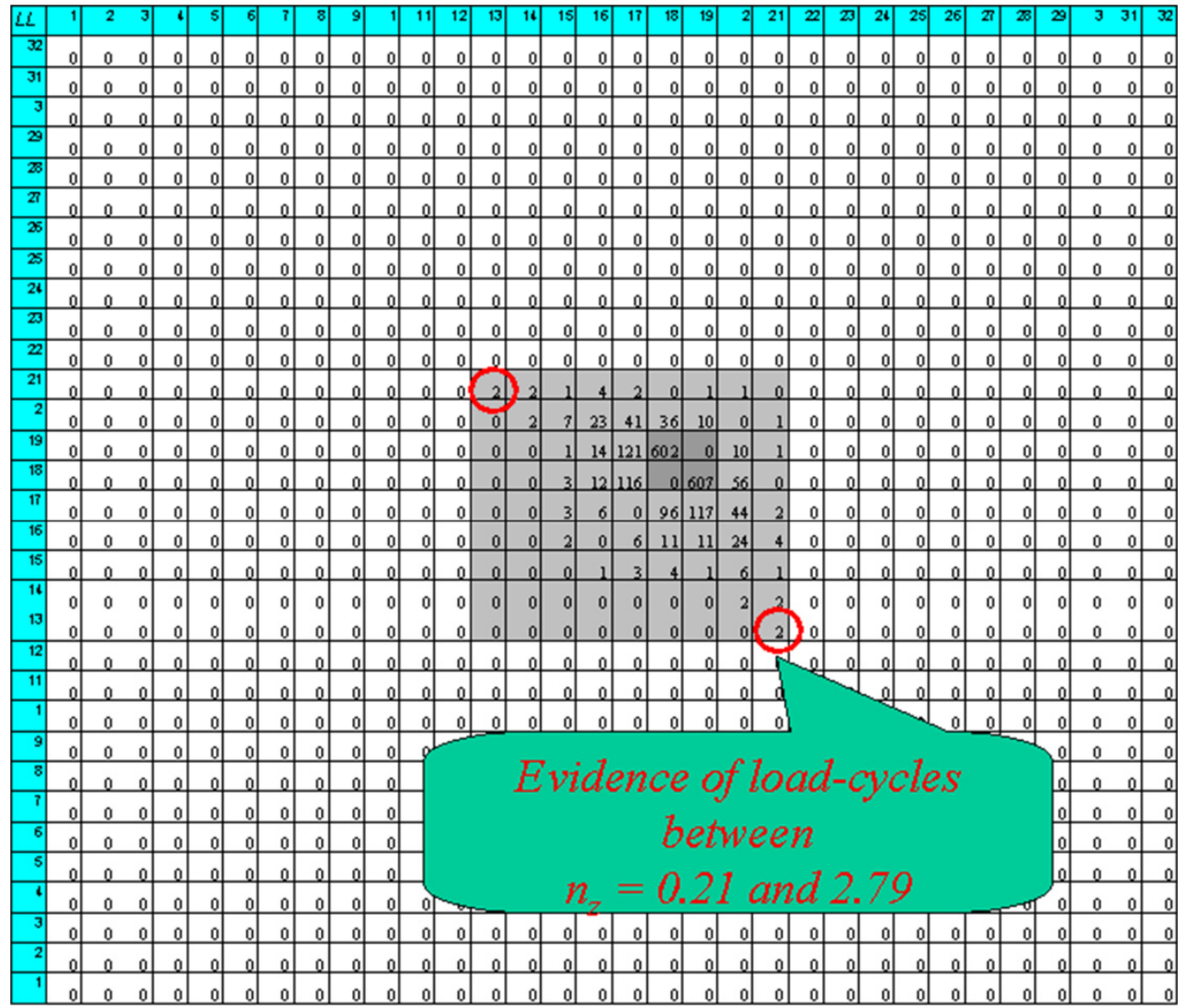

Fig. 11. HC-array from Flight No. 3 (autopilot control mode)

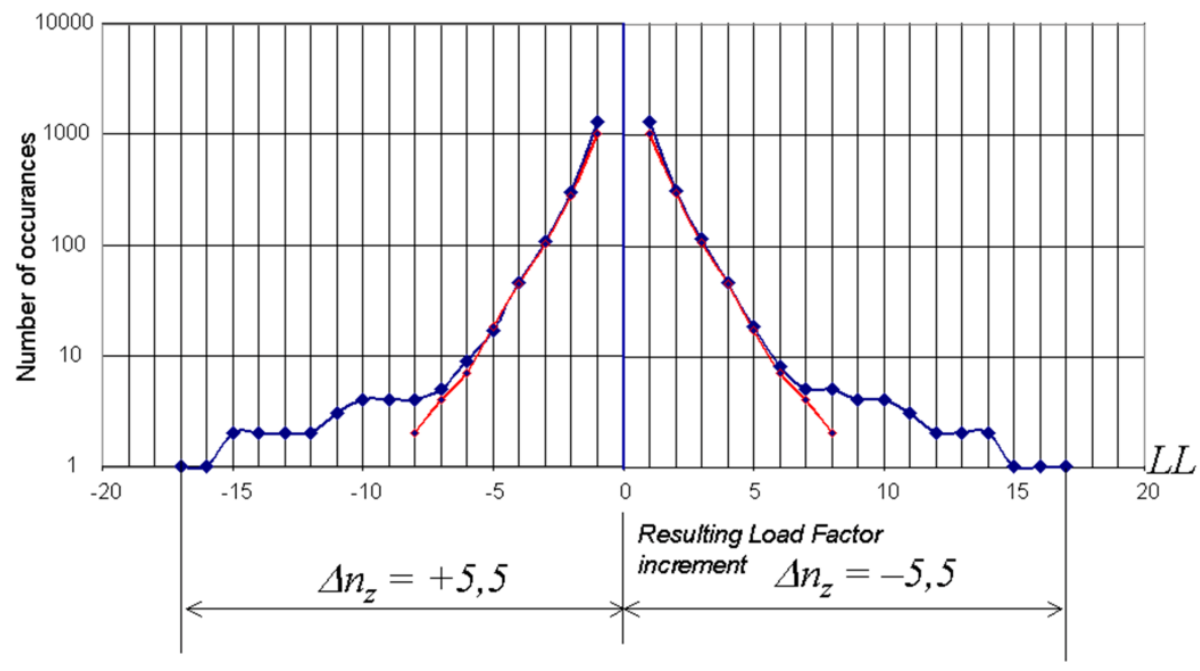

Fig. 12. Comparison of incremental load spectra generated by manual and autopilot control modes

Generally, to be able to determine effectively the UAV load spectrum it is necessary to increase the number of analyzed flights taking into consideration different scenarios, different weather and terrain conditions,, different pilots, etc. This would permit statistical analysis of occurrences of particular load increments. In the upper part of Fig. 13 there are two curves: the mean values and the values shifted up by 3 standard deviations. The latter curve takes into account possible dispersion of load increments occurrences (i.e. the number of appearances in relation to the flight times). On this basis it is possible to determine among other values the coefficients that should be applied for multiplication of the values existing in the $\mathbf{H C}$-array compound from all analyzed flights in order to obtain more generalized results. 


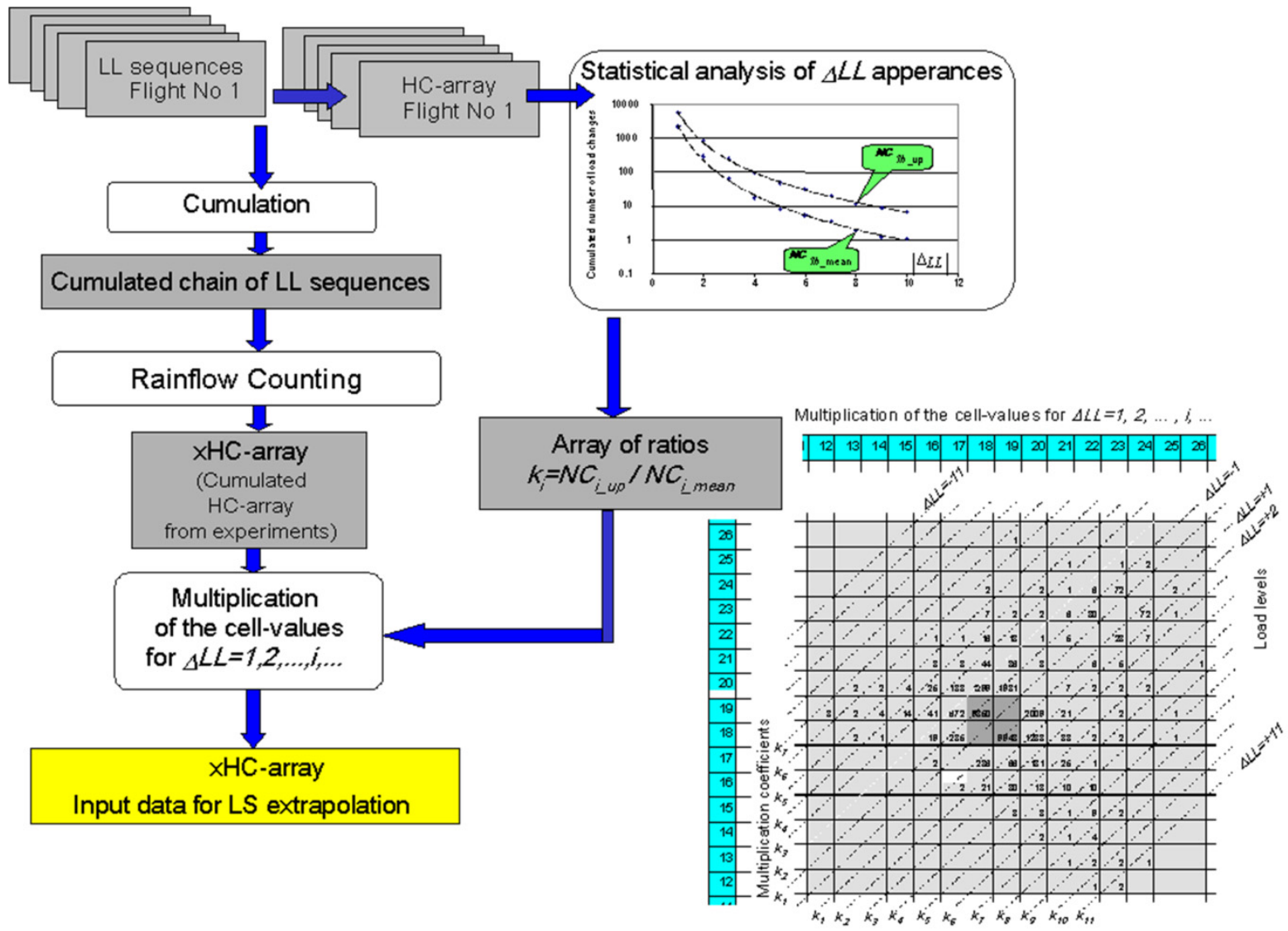

Fig. 13. Algorithm of data processing for model-load spectra-general case

\section{STRENGTH AND FATIGUE TESTING}

This chapter describes selected strength or fatigue tests for which load spectrum is of crucial importance. The first test concerns the spar of the UAV-SAMONIT1's wing (Fig. 14) made from CFRP composites.

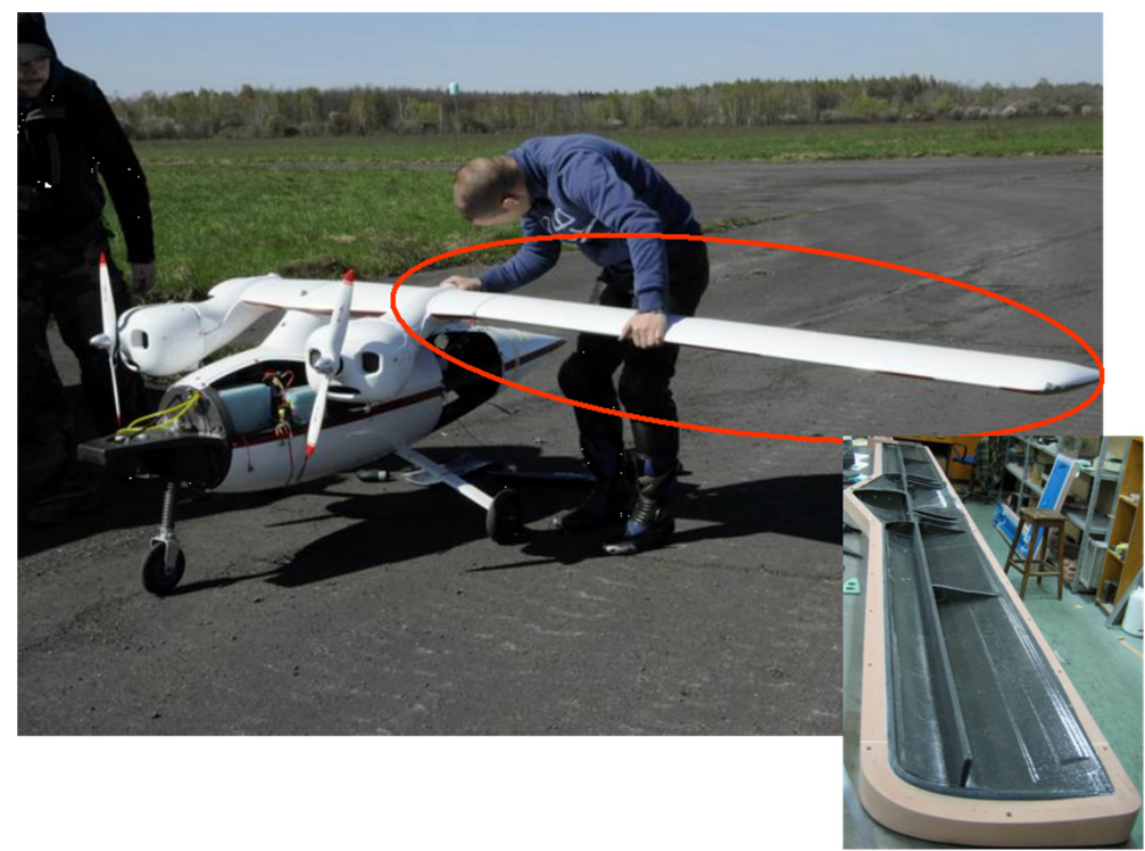

Fig. 14. CFRP-wing structure of SAMONIT1 
The main part of the wing structure is a wing spar. It can be tested together with the whole wing. For economical reasons, however, a simplified composite torsion tube is quite often used in testing instead of the real wing skins. An important aspect of fatigue investigations is frequent control of fatigue damage propagation by means of the NDT devices (Fig. 15).

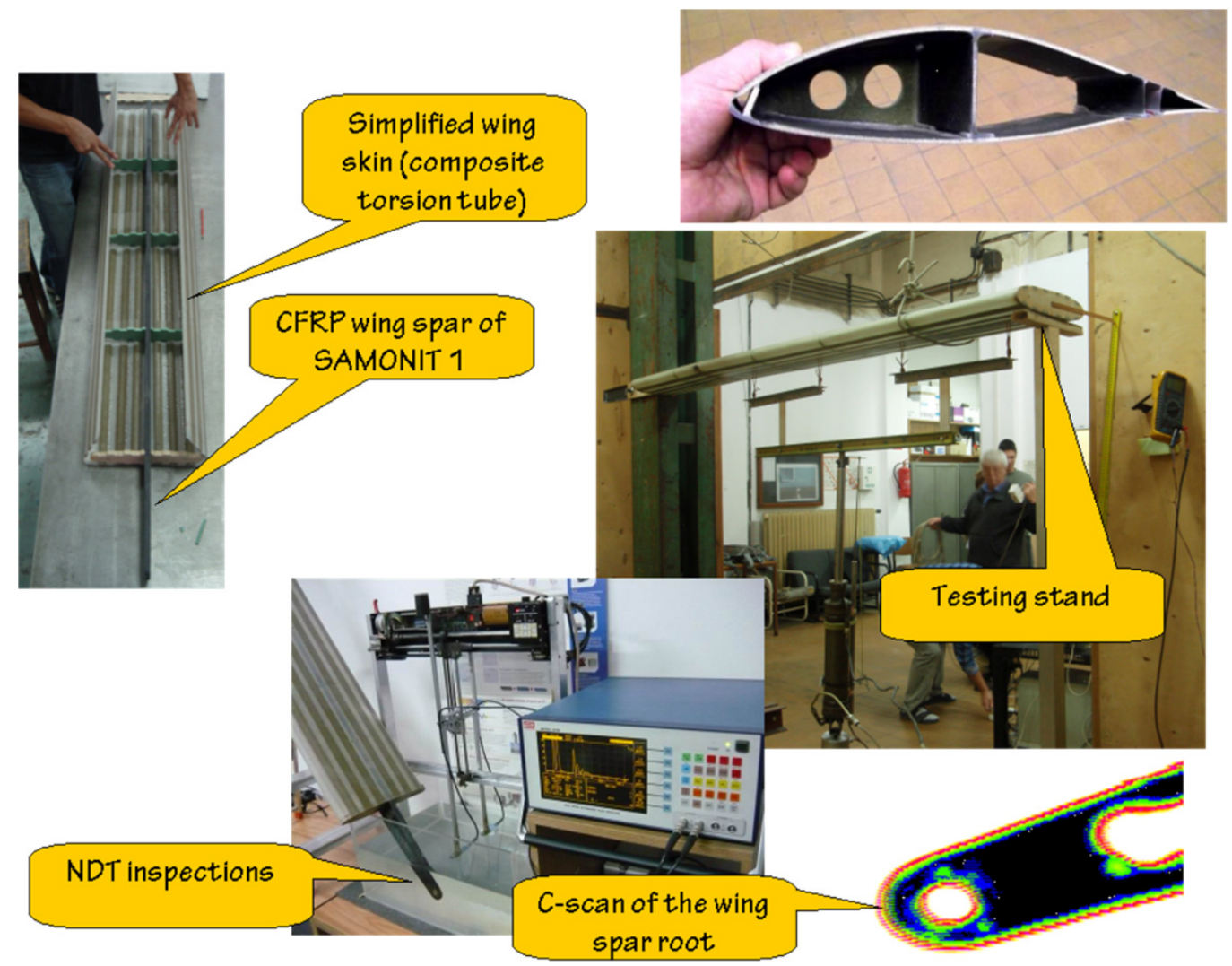

Fig. 15. Fatigue and NDT testing of SAMONIT 1's wing spar

Another element, which needs to be tested, is the tail beam, especially the joints between the beam and the aircraft structure (Fig. 16).

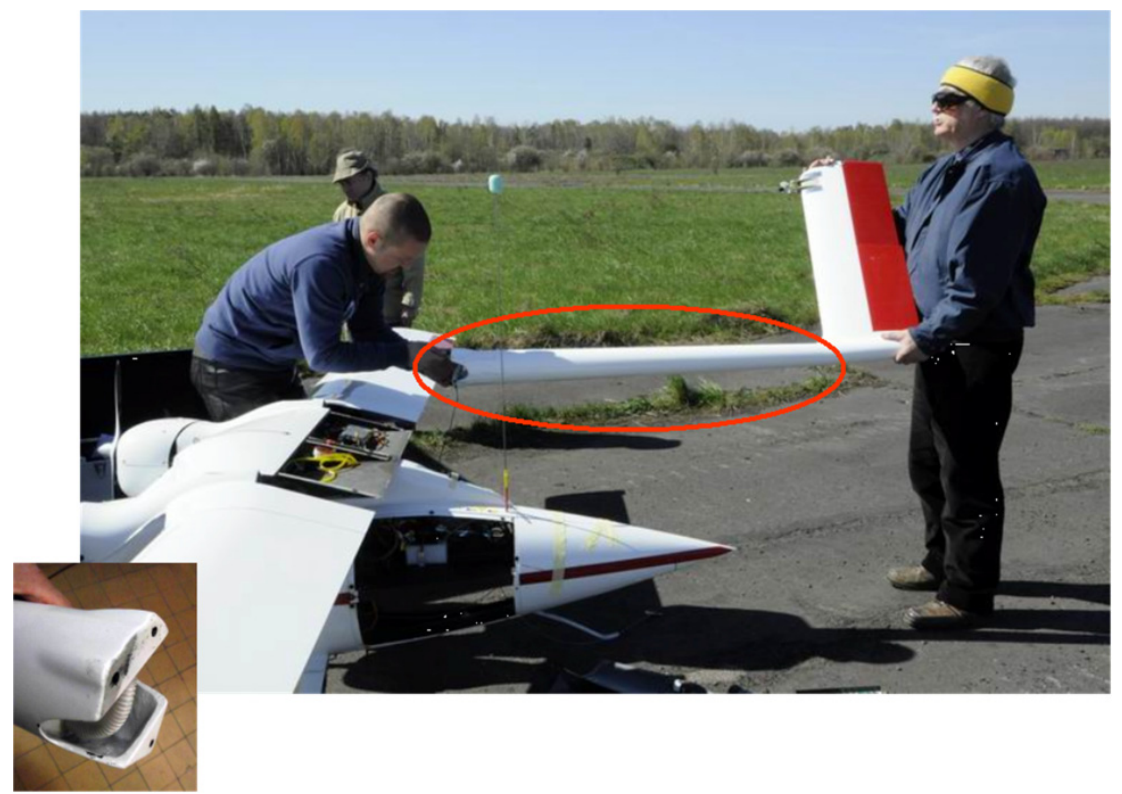

Fig. 16. Tail beam of SAMONIT 1 


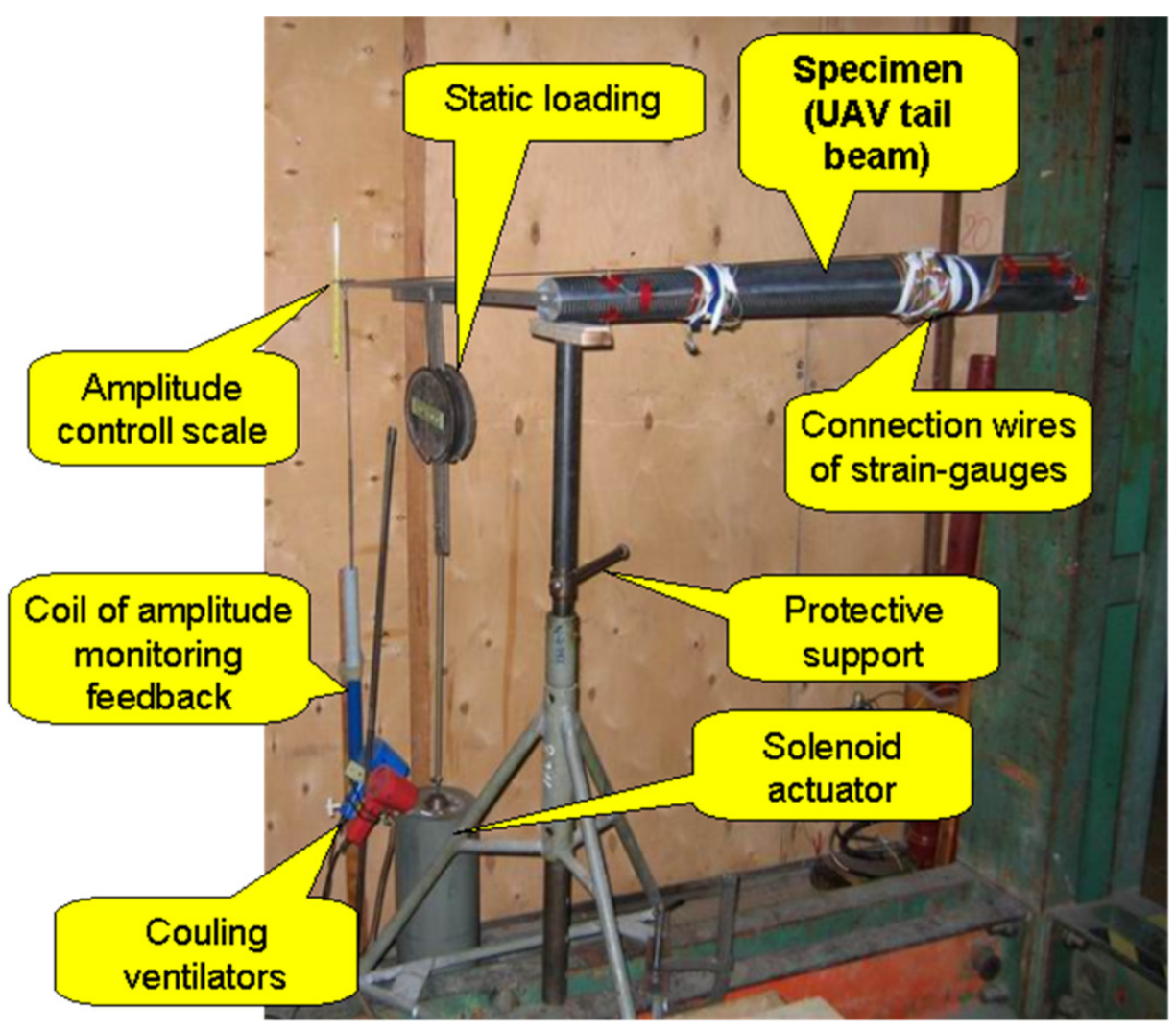

Fig. 17. Fatigue stand for UAV tail beam testing

Another crucial element of the UAV is the landing gear (Fig. 18). In comparison with GA aircraft this element is much more exposed to the damage risk because the UAV systems are not able to compensate for lack of the information normally accessible for the pilot seating in the cockpit.

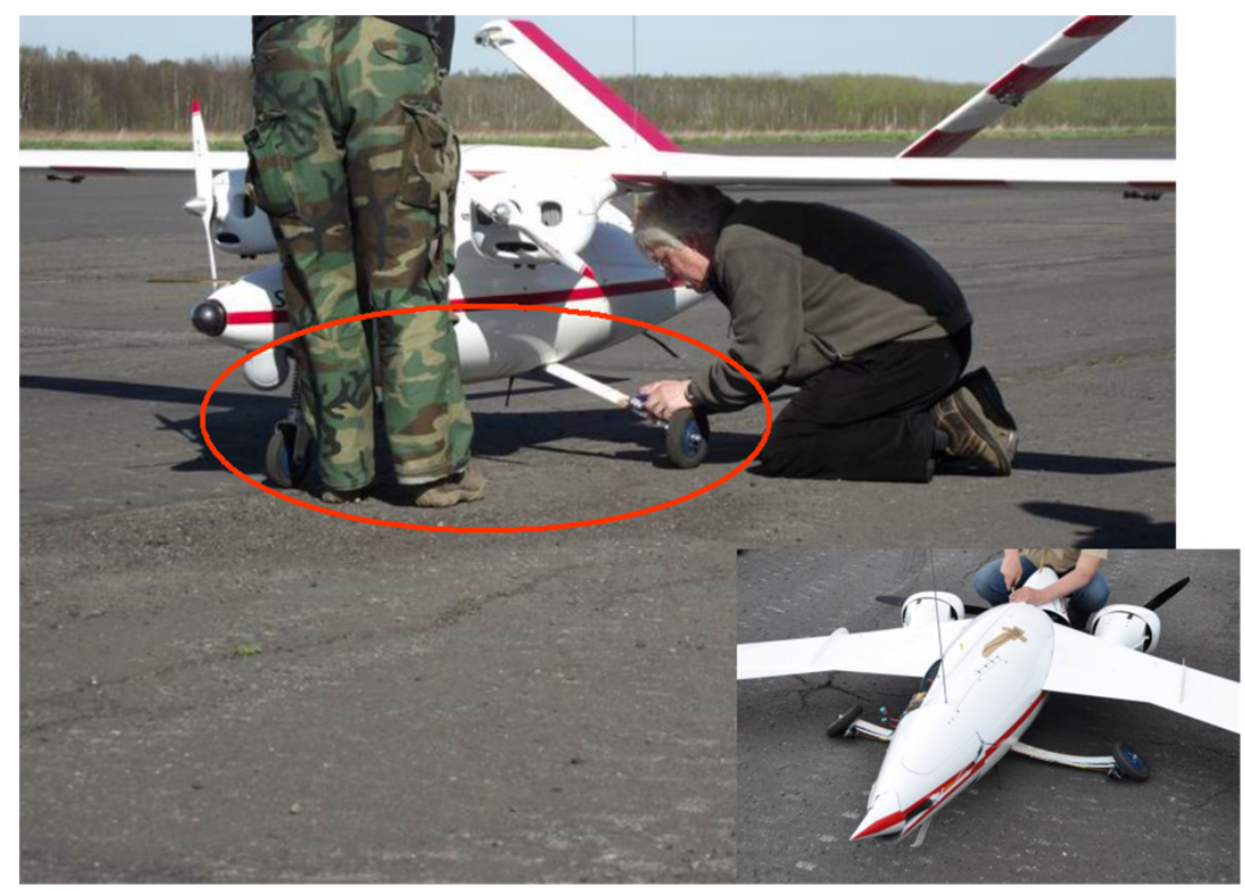

Fig. 18. Landing gear of SAMONIT 1 


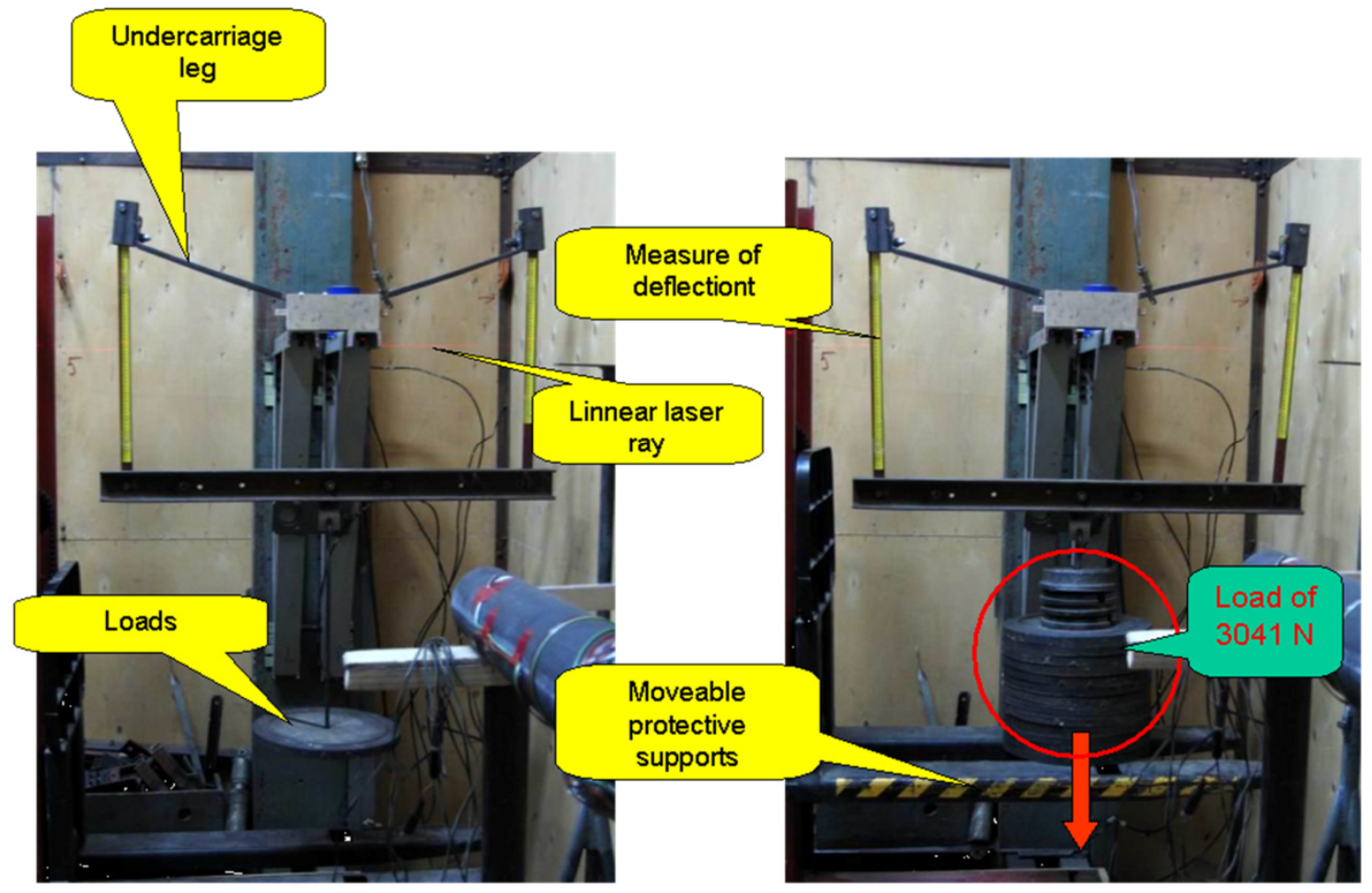

Fig. 19. Test-stand of UAV landing gear

\section{CONCLUSIONS}

- Determination of UAVs load spectra has a crucial importance for the process of elaborating airworthiness requirements for this category of aircraft.

- It was found that significant differences in load spectra could be observed between a manual and an automatic control mode of the UAV. Those differences may have strong influence on the fatigue life of the UAV structure.

- In order to determine airworthiness requirements for fatigue safety of the UAV structure it is necessary to increase the number of analyzed flights taking into consideration different flight scenarios, different weather and terrain conditions, different pilots, etc.

- Well-estimated load spectra and reliable fatigue tests are necessary to prove technical safety of UAVs.

\section{REFERENCES}

[1] Rodzewicz, M. Determination and extrapolation of the glider load spectra. Aircraft Engineering and Aerospace Technology, Vol. 80(5), pp. 487-496.

[2] Rodzewicz, M. Fatigue evaluation of high-loaded aeronautical composite structures. Aircraft Engineering and Aerospace Technology, Vol. 81(5), pp. 452-460.

[3] Rodzewicz M., Czerwiński G., Kubica B. J.: Load spectrum - archiving, analysis and load sequences reproduction (in Polish). Proceedings of XIII Conf. Mechanics in Aviation, PTMTS ML-XIII 2008, vol. 1, pp.43-55.

[3] Owczarek W., Rodzewicz M. (2009). Investigations into glider chassis load spectrum, Fatigue of Aircraft Structures Monographic Series, Issue 2009, pp. 150-170. 